\title{
Synchronization Analysis of General Complex Dynamical Networks with Couplings Delays and Delays in the Dynamical Nodes
}

\author{
Haiyi Sun \\ College of Science \\ Shenyang JianZhu University \\ Shenyang, China \\ shy_xx@163.com \\ Ning Li \\ Institute of Systems Science \\ Northeastern University \\ Shenyang, China \\ Lining80@163.com(corresponding author)
}

\author{
Zhongtang Chen \\ College of Science \\ Shenyang JianZhu University \\ Shenyang, China \\ Chen630609@163.com \\ Jinbao Wang \\ College of Science \\ Shenyang JianZhu University \\ Shenyang, China \\ jinbao_wang@163.com
}

\begin{abstract}
In this paper, we first introduce a more general model of complex dynamical networks with coupling delays and delays in the dynamical nodes than before. Then we further study the synchronization analysis of the complex dynamical networks with coupling delays and delays in the dynamical nodes. Via the theory of Lyapunov-Krasovskii stability and linear matrix inequality (LMI) technique, we investigate the sufficient conditions about synchronization criteria by constructing appropriate Lyapunov functions. The new delay-dependent conditions presented in the paper are formulated in the form of LMI, which can be solved easily by the LMI toolbox in Matlab. The node dynamic need not satisfy the very strong and the matrix is not assumed to be symmetric or irreducible. Moreover, the resulting for network synchronization are expressed in simple forms that can be readily applied in practical situations. The numerical example of the synchronization problem between the nonlinear electromechanical transducers has been investigated, which demonstrate the effectiveness of proposed results.
\end{abstract}

Keywords- complex networks; coupling delays; delayed nodes; synchronization; linear matrix inequality

\section{INTRODUCTION}

Complex networks have sprung up in recent 20 years, presently permeating in various fields of sciences, from physics science to biological science, even to society science[1-7]. A complex network is a large set of interconnected nodes, where the nodes and connections can represent everything. There have been a rich body of literature on analyzing complex networks, and one of the most significant dynamical behaviors of complex networks that has been widely investigated is the synchronization motion of its dynamical elements[1-3,7]. Some synchronization phenomena are very useful for us, such as the synchronous transfer of digital or analog signals in communication networks [8].
In practice, the information transmission within complex networks is in general not instantaneous since the signals traveling speed is limited, and this is very common in biological and physical networks [8-10].This fact gives rise to the time delays that may cause undesirable dynamic network behaviors such as oscillation and instability. Therefore, time delays should be modeled in order to simulating more realistic networks.

In this paper, we first introduce a more general model of complex dynamical network with coupling delays and delays in the dynamical nodes than before. Then we further study the synchronization of this model. Based on the theory of asymptotic stability of linear time-delay systems and Lyapunov method combined with linear matrix inequality technique, sufficient conditions about synchronization criteria are derived. It should be pointed out that the node dynamic need not satisfy the very strong and the matrix is not assumed to be symmetric or irreducible.

The rest of the paper is organized as follows. In Section 2 , the model of a general complex dynamical networks with coupling delays and delays in the dynamical nodes is presented and some preliminaries are also given. In Section 3, sufficient conditions about synchronization criteria of this complex networks is obtained. The numerical example for verifying the theoretical result is given in Section 4. Finally, conclusions are presented in Section 5.

\section{MODEL DESCRIPTION AND PRELIMINARIES}

The complex networks with coupling delays and delays in thedynamical nodes can be described as follows: 


$$
\begin{aligned}
\dot{x}_{i}(t)= & f\left(x_{i}(t), x_{i}\left(t-\tau_{1}\right)\right)+\sum_{j=1}^{N} a_{i j} \Gamma_{1} x_{j}(t) \\
& +\sum_{j=1}^{N} b_{i j} \Gamma_{2} x_{j}\left(t-\tau_{2}\right),(i=1,2, \cdots, N)
\end{aligned}
$$

where $x_{i}(t)=\left(x_{i 1}(t), x_{i 2}(t), \cdots, x_{i n}(t)\right)^{\mathbb{T}} \in R^{n}$ is the state and input variable of node $i$ at timet. $f: R^{n} \times R^{n} \rightarrow R^{n}$ is a continuous and differentiable function, $\tau_{1}$ and $\tau_{2}>0$ are the time delay of coupling delays and delays in the dynamical nodes, respectively, which are arbitrary but bounded, i.e., $\tau_{1}, \tau_{2} \in(0, h]$, where $h$ is a positive constant. $A=\left(a_{i j}\right) \in R^{N \times N}$ and $B=\left(b_{i j}\right) \in R^{N \times N}$ are the coupling matrices with zero-sum rows, which represent the coupling strength and the underlying topology for nondelayed configuration and delayed one $\tau_{2}$ at time $t$, respectively, $a_{i j} \geq 0, b_{i j} \geq 0$ for $i \neq j, a_{i j}, b_{i j}$ are defined as follows: if there is a connection from node $j$ to node $i(i \neq j) \quad a_{i j}>0, b_{i j}>0$, otherwise $a_{i j}=0, b_{i j}=0(i \neq j)$, and $\Gamma_{1}, \Gamma_{2} \in R^{n \times n}$ are positive diagonal matrices which describe the individual couplings between node $i$ and $j$ for non-delayed configuration and delayed one $\tau_{2}$ at time $t$ respectively.

When the delayed dynamical network (1) achieves synchronization, namely, the states $x_{1}(t) \rightarrow x_{2}(t) \rightarrow \cdots \rightarrow$ $x_{N}(t) \rightarrow s(t)$, as $t \rightarrow \infty$, where $s(t) \in R^{n}$ is a solution of an isolate node, i.e.

$$
\dot{s}(t)=f\left(s(t), s\left(t-\tau_{1}\right)\right) .
$$

$s(t)$ can be an equilibrium point, a nontrivial periodic orbit, or even a chaotic orbit. Let $C\left([-h, 0], R^{n}\right)$ be the Banach space of continuous functions mapping the interval $[-h, 0]$ into $R^{n}$ with the norm $\|\phi\|=\sup _{-h \leq \theta \leq 0}\|\phi(\theta)\|$, where $\|\cdot\|$ is the Euclidean norm. The rigorous mathematical definition of synchronization for delayed dynamical network (1) is introduced as follows.

Definition 1. Let $x_{i}\left(t ; t_{0} ; \phi\right), i=1,2, \cdots, N$ be a solution of delayed dynamical network (1), where $\phi=\left(\phi_{1}^{\mathrm{T}}, \phi_{2}^{\mathrm{T}}, \cdots \phi_{N}^{\mathrm{T}}\right)^{\mathrm{T}}, \phi_{i}=\phi_{i}(\theta) \in C\left([-h, 0], R^{n}\right)$ are initial conditions. If there is a nonempty subset $\Lambda \subseteq R^{n}$, such that $\phi_{i}$ take values in $\Lambda$ and $x_{i}\left(t ; t_{0} ; \phi\right) \in R^{n}$ for all $t \geq t_{0}$ and

$$
\lim _{t \rightarrow \infty}\left\|x_{i}\left(t ; t_{0} ; \phi\right)-s\left(t ; t_{0} ; s_{0}\right)\right\|=0, \quad i=1,2, \cdots, N
$$

where $s\left(t ; t_{0} ; s_{0}\right)$ is a solution of the system (2) with $s_{0} \in R^{n}$, then the delayed dynamical network (1) is said to realize synchronization, and $\Lambda \times \Lambda \times \cdots \times \Lambda$ is called the region of synchrony of the delayed dynamical network (1).

Define the error vector by

$$
e_{i}(t)=x_{i}(t)-s(t), \quad i=1,2, \cdots, N
$$

Notice that in (1) $\sum_{j=1}^{N} a_{i j}=0$ and $\sum_{j=1}^{N} b_{i j}=0$, then the error system can be described by

$$
\begin{aligned}
\dot{e}_{i}(t)= & f\left(x_{i}(t), x_{i}\left(t-\tau_{1}\right)\right)-f\left(s(t), s\left(t-\tau_{1}\right)\right) \\
& +\sum_{j=1}^{N} a_{i j} \Gamma_{1} e_{j}(t)+\sum_{j=1}^{N} b_{i j} \Gamma_{2} e_{j}\left(t-\tau_{2}\right) .
\end{aligned}
$$

Then the synchronization problem of the dynamical network (1) is equivalent to the problem of stabilization of the error dynamical system (5).

Assumption 1. (A1) Let $S=\operatorname{diag}\left(\beta_{1}, \beta_{2}, \cdots, \beta_{N}\right)$ be a positive-definite diagonal matrix. The nonlinear vectorvalued continuous function, $f: R^{n} \times R^{n} \times R^{+} \rightarrow R^{n} \quad$ satisfied the semi-lipschiz condition:

$$
\left(x_{i}(t)-s(t)\right)^{\mathrm{T}} S\left(f\left(x_{i}(t), x_{i}\left(t-\tau_{1}\right)\right)-f\left(s(t), s\left(t-\tau_{1}\right)\right)\right)
$$$$
\leq \gamma_{1}(t)\left\|x_{i}(t)-s(t)\right\|^{2}+\gamma_{2}(t)\left\|x_{i}\left(t-\tau_{1}\right)-s\left(t-\tau_{1}\right)\right\|^{2} .
$$

where $i=1,2, \cdots, N, \gamma_{1}(t)$ and $\gamma_{2}(t)$ are unknown timevarying nonzero parameters with unknown bounds, that is $\gamma_{1}(t) \in\left[\underline{\gamma_{1}}, \overline{\gamma_{1}}\right]$ and $\gamma_{2}(t) \in\left[\underline{\gamma_{2}}, \overline{\gamma_{2}}\right] . \underline{\gamma_{1}}, \overline{\gamma_{1}}, \underline{\gamma_{2}}, \overline{\gamma_{2}}$, are unknown constants.

We define $\gamma_{1}=\max \left(\underline{\gamma_{1}}, \overline{\gamma_{1}}\right)$ and $\gamma_{2}=\max \left(\underline{\gamma_{2}}, \overline{\gamma_{2}}\right)$.

Note that Assumption 1 is less conservative than general uniformly Lipschitz condition. For example, all linear and piecewise linear functions satisfy this condition. In addition, if $\frac{\partial f_{i}}{\partial x_{j}}(i, j=1,2, \cdots, n)$ are bounded and $\Gamma_{0}$ is positive definite, the above condition is satisfied. So, it includes many well-known systems, such as the Lorenz system, Chen system, Lü system, recurrent neural networks, Chua's circuit, and so on.

Lemma 1. For any vectors $x, y \in R^{m}$ positive definite matrix $Q \in R^{m \times m}$, the following matrix inequality holds:

$$
2 x^{\mathrm{T}} y \leq x^{\mathrm{T}} Q x+y^{\mathrm{T}} Q^{-1} y
$$

If not specified otherwise, inequality $Q>0$ ( $Q<0, Q \geq 0, Q \leq 0$ )means $Q$ is a positive (or negative, or semi-positive, or semi-negative) definite matrix, where $Q$ is a square matrix.

Lemma 2. The Kronecker product ' $\otimes$ ' have the following properties:

(1) $(\alpha A) \otimes B=A \otimes(\alpha B)$,

(2) $(A+B) \otimes C=A \otimes B+A \otimes C$,

(3) $(A \otimes B)(C \otimes D)=(A C) \otimes(B D)$,

(4) $(A \otimes B)^{\mathrm{T}}=A^{\mathrm{T}} \otimes B^{\mathrm{T}}$.

\section{SYNCHRONIZATION CRITERIA OF THE GENERAL DELAYED COMPLEX DYNAMICAL NETWORKS}

In this section, we are in the position to present our main results for synchronization criteria of the general complex dynamical networks with couplings delays and delays in the dynamical nodes. 
Let $F=\left(f\left(x_{1}(t), x_{1}\left(t-\tau_{1}\right)\right)-f\left(s(t), s\left(t-\tau_{1}\right)\right), \cdots, f\left(x_{N}(t)\right.\right.$, $\left.\left.x_{N}\left(t-\tau_{1}\right)\right)-f\left(s(t), s\left(t-\tau_{1}\right)\right)\right), \bar{A}=A \otimes \Gamma_{1}, \bar{B}=B \otimes \Gamma_{2}$, $e(t)=\left(e_{1}^{\mathrm{T}}(t), e_{2}^{\mathrm{T}}(t), \cdots, e_{N}^{\mathrm{T}}(t)\right)^{\mathrm{T}}, u(t)=\left(u_{1}^{\mathrm{T}}(t), u_{2}^{\mathrm{T}}(t), \cdots, u_{N}^{\mathrm{T}}(t)\right)^{\mathrm{T}}$.

With the Kronecker product ' $\otimes$ ' for matrices, system (5) can be recast into

$$
\dot{e}(t)=I_{n N} F+\bar{A} e_{j}(t)+\bar{B} e_{j}\left(t-\tau_{2}\right)+u(t),
$$

Based on a Lyapunov-Krasovskii function, we first detrude the synchronization criteria of the network (1) with no input variables of node $i$ (i.e., $\left.u_{i}(t)=0\right)$.

Theorem 1. Consider the complex networks (1) with no input variables $\tau_{1}, \tau_{2} \in(0, h]$ and no input variables. If there exist matrices $P>0, Q_{1}>0, Q_{2}>0, R_{1}>0$, $R_{2}>0$ and any matrices $N_{i}, M_{i}, \quad T_{i}(i=1, \cdots, 5)$ of appropriate dimensions such that the following LMI holds: $\left[\begin{array}{lllllllll}\Pi_{11} & \Pi_{12} & \Pi_{13} & \Pi_{14} & \Pi_{15} & \Pi_{16} & \Pi_{17} & \Pi_{18} & \Pi_{19} \\ * & \Pi_{22} & \Pi_{23} & \Pi_{24} & \Pi_{25} & \Pi_{26} & \Pi_{27} & \Pi_{28} & \Pi_{29} \\ * & * & \Pi_{33} & \Pi_{34} & \Pi_{35} & \Pi_{36} & \Pi_{37} & \Pi_{38} & \Pi_{39} \\ * & * & * & \Pi_{44} & \Pi_{45} & \Pi_{46} & \Pi_{47} & \Pi_{48} & \Pi_{49} \\ * & * & * & * & \Pi_{55} & \Pi_{56} & \Pi_{57} & \Pi_{58} & \Pi_{59} \\ * & * & * & * & * & \Pi_{66} & \Pi_{67} & \Pi_{68} & \Pi_{69} \\ * & * & * & * & * & * & \Pi_{77} & \Pi_{78} & \Pi_{79} \\ * & * & * & * & * & * & * & \Pi_{88} & \Pi_{89} \\ * & * & * & * & * & * & * & * & \Pi_{99}\end{array}\right]<0$.

where

$$
\begin{aligned}
& \Pi_{11}=R_{1}+R_{2}+N_{1}+N_{1}^{\mathrm{T}}+T_{1}+T_{1}^{\mathrm{T}}+M_{1} \bar{A}+\bar{A}^{\mathrm{T}} M_{1}^{\mathrm{T}}, \\
& \Pi_{12}=-N_{1}+N_{2}^{\mathrm{T}}+T_{2}^{\mathrm{T}}+\bar{A}^{\mathrm{T}} M_{2}^{\mathrm{T}}, \\
& \Pi_{13}=N_{3}^{\mathrm{T}}-M_{1}+\bar{A}^{\mathrm{T}} M_{3}^{\mathrm{T}}+P+T_{3}^{\mathrm{T}}, \\
& \Pi_{15}=N_{5}^{\mathrm{T}}+T_{5}^{\mathrm{T}}-T_{1}+\bar{A}^{\mathrm{T}} M_{5}^{\mathrm{T}}+M_{1} \bar{B}, \Pi_{16}=h N_{1}, \\
& \Pi_{17}=h T_{1}, \Pi_{18}=\Pi_{29}=I, \Pi_{22}=-R_{1}-N_{2}-N_{2}^{\mathrm{T}}, \\
& \Pi_{23}=-N_{3}^{\mathrm{T}}-M_{2}, \quad \Pi_{24}=-N_{4}^{\mathrm{T}}+M_{2}, \\
& \Pi_{25}=-N_{5}^{\mathrm{T}}-T_{2}+M_{2} \bar{B}, \Pi_{26}=h N_{2}, \quad \Pi_{27}=h T_{2}, \\
& \Pi_{33}=h Q_{1}+h Q_{2}-M_{3}-M_{3}^{\mathrm{T}}, \Pi_{34}=M_{3}-M_{4}^{\mathrm{T}}, \\
& \Pi_{35}=-T_{3}-M_{5}^{\mathrm{T}}+M_{3} \bar{B} \Pi_{36}=h N_{3}, \quad \Pi_{37}=h T_{3}, \\
& \Pi_{44}=M_{4}+M_{4}^{\mathrm{T}}, \Pi_{45}=-T_{4}+M_{5}^{\mathrm{T}}+M_{4} \bar{B}, \\
& \Pi_{46}=h N_{4}, \quad \Pi_{47}=h T_{4}, \\
& \Pi_{55}=-R_{2}-T_{5}-T_{5}^{\mathrm{T}}+M_{5} \bar{B}+\bar{B}^{\mathrm{T}} M_{5}^{\mathrm{T}}, \quad \Pi_{56}=h N_{5}, \\
& \Pi_{57}=h T_{5}, \quad \Pi_{66}=-h Q_{1}, \quad \Pi_{77}=-h Q_{2}, \quad \bar{S}=S \otimes I_{n}, \\
& \Pi_{19}=\Pi_{28}=\Pi_{38}=\Pi_{39}=\Pi_{48}=\Pi_{49}=\Pi_{58}=\Pi_{59}=\Pi_{67}= \\
& \Pi_{68}=\Pi_{69}=\Pi_{78}=\Pi_{79}=\Pi_{89}=\Pi_{98}=0 .
\end{aligned}
$$

then the synchronization is achieved. * denotes the elements below the main diagonal of a symmetric block matrix.

Proof: Selecting a Lyapunov-Krasovskii function of the form

$$
\begin{aligned}
V(t)= & e^{\mathrm{T}}(t) P e(t)+\sum_{i=1}^{2} \int_{t-\tau_{i}}^{t} e^{\mathrm{T}}(s) R_{i} e(s) \mathrm{d} s+ \\
& \sum_{i=1}^{2} \int_{t-\tau_{i}}^{t} \int_{s}^{t} \dot{e}^{\mathrm{T}}(s) Q_{i} \dot{e}(s) \mathrm{d} v \mathrm{~d} s .
\end{aligned}
$$

Taking the time derivative of $V(t)$ along the trajectory of (7) yield that

$$
\begin{aligned}
& \dot{V}(t)= 2 e^{\mathrm{T}}(t) P \dot{e}(t)-\sum_{i=1}^{2} \int_{t-\tau_{i}}^{t} \dot{e}^{\mathrm{T}}(s) Q_{i} \dot{e}(s) \mathrm{d} s \\
&+\sum_{i=1}^{2} \tau_{i} \dot{e}^{\mathrm{T}}(t) Q_{i} \dot{e}(t)-\sum_{i=1}^{2} e^{\mathrm{T}}\left(t-\tau_{i}\right) R_{i} e\left(t-\tau_{i}\right) \\
&+\sum_{i=1}^{2} e^{\mathrm{T}}(t) R_{i} e(t)+2 \xi^{\mathrm{T}} N\left[e(t)-e\left(t-\tau_{1}\right)\right. \\
&\left.\quad-\int_{t-\tau_{1}}^{t} \dot{e}^{\mathrm{T}}(s) \mathrm{d} s\right]+2 \xi^{\mathrm{T}} T\left[e(t)-e\left(t-\tau_{2}\right)\right. \\
&\left.-\int_{t-\tau_{2}}^{t} \dot{e}^{\mathrm{T}}(s) \mathrm{d} s\right]+2 \xi \xi^{\mathrm{T}} M[F+\bar{A} e(t) \\
&\left.+\bar{B} e\left(t-\tau_{2}\right)-\dot{e}(t)\right]
\end{aligned}
$$

From Lemma 1, it follows that

$-2 \xi^{\mathrm{T}} N \int_{t-\tau_{1}}^{t} \dot{e}^{\mathrm{T}}(s) d s \leq h \xi^{\mathrm{T}} N Q_{1}^{-1} N^{\mathrm{T}} \xi+\int_{t-\tau_{1}}^{t} \dot{e}^{\mathrm{T}}(s) Q_{1} \dot{e}(s) \mathrm{d} s$,

$-2 \xi^{\mathrm{T}} T \int_{t-\tau_{2}}^{t} \dot{e}^{\mathrm{T}}(s) d s \leq h \xi^{\mathrm{T}} T Q_{2}^{-1} T^{\mathrm{T}} \xi+\int_{t-\tau_{2}}^{t} \dot{e}^{\mathrm{T}}(s) Q_{2} \dot{e}(s) \mathrm{d} s$,

Then, combining (10\})-(11) and A1, we have

$$
\begin{aligned}
\dot{V}(t) \leq & 2 e^{\mathrm{T}}(t) P \dot{e}(t)+\sum_{i=1}^{2}\left[h \dot{e}^{\mathrm{T}}(t) Q_{i} \dot{e}(t)+e^{\mathrm{T}}(t) R_{i} e(t)\right. \\
& \left.-e^{\mathrm{T}}\left(t-\tau_{i}\right) R_{i} e\left(t-\tau_{i}\right)\right]+h \xi^{\mathrm{T}} N Q_{1}^{-1} N^{\mathrm{T}} \xi \\
& +h \xi^{\mathrm{T}} T Q_{2}^{-1} T^{\mathrm{T}} \xi+2 \xi^{\mathrm{T}} N\left[e(t)-e\left(t-\tau_{1}\right)\right] \\
& +2 \xi^{\mathrm{T}} M\left[F+\bar{A} e(t)+\bar{B} e\left(t-\tau_{2}\right)-\dot{e}(t)\right] \\
& +2 \xi^{\mathrm{T}} T\left[e(t)-e\left(t-\tau_{2}\right)\right]+\gamma_{1} e^{\mathrm{T}}(t) e(t) \\
& +\gamma_{2} e^{\mathrm{T}}\left(t-\tau_{1}\right) e\left(t-\tau_{1}\right)-e^{\mathrm{T}}(t) \bar{S} F \\
= & \xi^{\mathrm{T}} \Omega
\end{aligned}
$$

where

$$
\begin{aligned}
\Omega= & {\left[\begin{array}{ccccc}
\Pi_{11}+\gamma_{1} I & \Pi_{12} & \Pi_{13} & \Pi_{14} & \Pi_{15} \\
* & \Pi_{22}+\gamma_{2} I & \Pi_{23} & \Pi_{24} & \Pi_{25} \\
* & * & \Pi_{33} & \Pi_{34} & \Pi_{35} \\
* & * & * & \Pi_{44} & \Pi_{45} \\
* & * & * & * & \Pi_{55}
\end{array}\right] } \\
& +h N Q_{1}^{-1} N^{\mathrm{T}}+h T Q_{2}^{-1} T^{\mathrm{T}},
\end{aligned}
$$

where $\Pi_{i j}(i, j=1, \cdots, 5)$ are given by $(8)$.

By Schur complement and matrix inequality (8), we can get $\Omega<0$. That is $\dot{V}<0$. From (8), it is guaranteed that all the subsystems in (1) are synchronized for any fixed time delay $\tau \in(0, h]$. This completes the proof.

Remark 1. Base on LMI method, we construct a more general Lyapunov function to analyze the synchronization problem of the complex dynamical networks with couplings delays and delays in the dynamical nodes. The new delay-dependent conditions presented in Theorem 1 
are formulated in the form of LMI, which can be solved by the LMI toolbox in Matlab.

\section{NUMERICAL SIMULATIONS}

Example 1. We show that a delayed network with $N=5$ nodes described by (14). Consider a delayed electromechanical device network as the node dynamical system. It is composed of an electrical part (Duffing oscillator) coupled to a mechanical part governed by a linear oscillator. The coupling between both parts is realized through the electromagnetic force due to a permanent magnet. It creates a Laplace force in the mechanical part and the Lenz electromotive voltage in the electrical part. The electrical part of the system consists of a resistor $R$, an inductor $L$, a condenser $C$ and a sinusoidal voltage source $e(t)$ all connected in series. The mechanical part is composed of a mobile beam which can move along the $\vec{z}$-axis on both sides. The rod T which has the similar motion is bound to a mobile beam with a spring. A single delayed dynamical equation is described by the following form[11]:

$$
f=\left\{\begin{array}{l}
35\left(x_{i 2}(t)-x_{i 1}(t)\right), \\
-7 x_{i 1}(t)-x_{i 1}(t) x_{i 3}\left(t-\tau_{1}\right)+28 x_{i 2}(t), \\
x_{i 1}(t) x_{i 2}\left(t-\tau_{1}\right)-3 x_{i 3}\left(t-\tau_{1}\right) .
\end{array}\right.
$$

where the coupling matrices are

$$
A=\left(\begin{array}{ccccc}
-1 & \frac{1}{4} & \frac{1}{4} & \frac{1}{4} & \frac{1}{4} \\
\frac{1}{2} & -1 & 0 & \frac{1}{2} & 0 \\
0 & 1 & -1 & 0 & 0 \\
\frac{1}{3} & 0 & \frac{1}{3} & -1 & \frac{1}{3} \\
0 & \frac{1}{2} & \frac{1}{2} & 0 & -1
\end{array}\right),
$$

$\tau_{1}, \tau_{2} \in(0,1.2]$. The individual couplings matrices are $\Gamma_{1}=\Gamma_{2}=\operatorname{diag}\{1,1,1\}$.Similar to [12], obviously, assumptions A1 holds.

Applying Theorem 1 with, $\gamma_{1}=\gamma_{2}=4, \tau_{1}=0.6$, $\tau_{2}=1.2$ and solving the LMI (8) by using LMI toolbox of Matlab, it is found the LMI (8) is feasible. The synchronous error $e_{i}$ is shown in Fig .1. For this simulation, the initial values of states are $x_{i}(0)=(2,2,3)^{\mathrm{T}}$ and $s(0)=(1,1,1)^{\mathrm{T}}$.
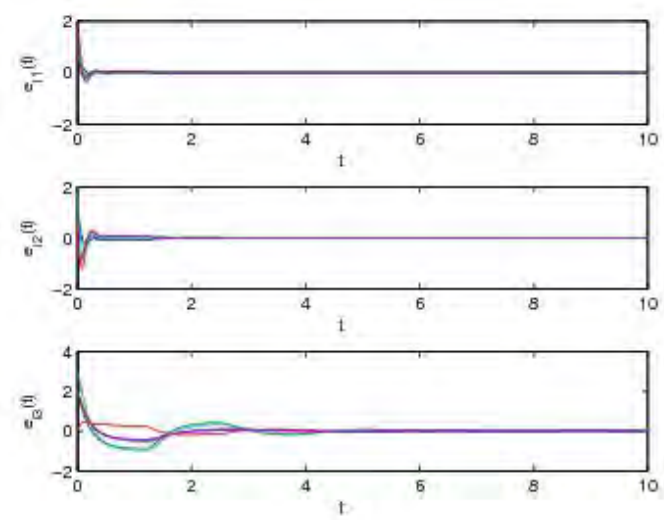

Figure 1. Synchronization errors $e_{i 1}, e_{i 2}, e_{i 3}(i=1,2, \cdots, 5)$ of networks (12).

\section{CONCLUSION}

A general complex dynamical network with coupling delays and delays in the dynamical nodes which represents a realistic form of networks has been studied in this paper. We investigate the sufficient conditions about synchronization criteria via the theory of Lyapunov stability and linear matrix inequality technique. By constructing appropriate Lyapunov functions, the synchronization criteria are derived. Moreover, the resulting for network synchronization are expressed in simple forms that can be readily applied in practical situations. Finally, the effectiveness of these synchronization criteria are verified by numerical simulations.

\section{ACKNOWLEDGMENT}

This work was partially supported by the Science Research Project of Liaoning Provincial Education Department (grant no. L2012208), the Science Foundation of Ministry of Housing and Urban-Rural Development (grant no. 2013-K5-2) and National Science Foundation of Shenyang Jianzhu University (2013172,2013174).

\section{REFERENCES}

[1] X.F. Wang, X. Li, and G.R. Chen, "Complex network theory and its application”, Beijing: Tsinghua University press, 2006.

[2] D.W. Gong, H.G., Zhang, B.N. Huang, and Z.Y. Ren, "Synchronizationcriteria and pinning control for complex networks with multipledelays", Neural Comput . Appl., vol.22, pp. 151-159, 2013

[3] J.H. Lü and G.R. Chen, "A time-varying complex dynamical network model and its controlled synchronization criteria", IEEE Trans. Autom. Control, vol. 50, pp. 841-846, 2005.

[4] R. Albert and A.L. Barab'asi, "Statistical mechanices of complex networks", Rev. Mod. Phys., vol. 74, pp. 47-97, 2002.

[5] S.H. Strogatz, "Exploring complex networks", Nature, vol. 410, pp. 268-276, 2001.

[6] H.Y. Sun, Q.L. Zhang and N. Li, "Synchronization control of united complex dynamical networks with multi-links", Int. J. Innov. Comput. Inf. Control, vol. 7, pp. 927-939, 2011.

[7] H.Y. Sun, Q.L. Zhang and N. Li, "Synchronization of delayed complexdynamical networks via adaptive periodically intermittent control", Control and Decis., vol.28, pp. 797-800, 2013

[8] T. Liao and S. Tsai, "Adaptive synchronization of chaotic systems and its application to secure communications", Chaos, Solitons \& Fractals, vol. 11, pp. 1387-1396, 2000.

[9] H.Y. Sun, Q.L. Zhang, N. Li and H. Niu, "Pinning synchronization of directed complex dynamical networks with multilinks", The 
Third International Workshop on Chaos-Fractals Theories and Applications, 2010.10, Kunming: pp.24-28.

[10] N. Li, H.Y. Sun, X. Jing, and Q.L. Zhang, "Exponential synchronisation of united complex dynamical networks with multilinks via adaptive periodically intermittent control", IET Control Theory Appl., vol. 7, pp. 1725-1736, 2013
[11] D.M. Li, J.A. Lu, X.Q. Wu and G.R. Chen, "Estimating the ultimate bound and positively invariant set for the Lorenz system and a unified chaotic system", J. Math. Anal. Appl., vol. 323, pp. 844-853, 2006.

[12] R. Yamapi, J.B. Chabi Orou and P. Woafo, "Harmonic oscillations, stability and chaos control in a non-linear electromechanical system", J. Sound Vibr, vol. 259, pp. 1253-1264, 2003. 